\section{Grünes Licht für Forschung und Entwicklung}

In Kooperation mit der Hamburger Roxall Medizin $\mathrm{GmbH}$ hatte das Unternehmen Dr. Beckmann Pharma im Jahr 2006 verschiedene moderne Präparate für die spezifische Immuntherapie in Deutschland eingeführt: Roxoid ${ }^{\circledR}$, Clustoid $^{\circledR}$, Sulgen ${ }^{\circledR}$ und Deposit ${ }^{\circledR}$. Inzwischen sind mit diesen Präparaten bereits mehr als 13.000 Patienten behandelt worden. Damit gehören die beiden Kooperationspartner zu den AllergenpräparateAnbietern mit den höchsten Zuwachsraten. Auch der Adrenalin Autoinjektor Anapen ${ }^{\circledR}$ ist für etliche Allergologen das Notfallmedikament der ersten Wahl für Patienten, die durch Anaphylaxie gefährdet sind.

Derzeit schafft das Unternehmen aus Seefeld bei München die Voraussetzungen für eine zukunftsweisende Forschung und Entwicklung im Bereich der Allergologie: Mit Dr. Enrique Fernández Caldas konnte dazu ein international renommierter Forschungsleiter gewonnen werden. Es sollen jetzt sowohl Forschungsprojekte im eignen Haus als auch in Kooperation mit Universitäten und Forschungsinstituten vorangetrieben werden.

Nach Informationen von Dr. Beckmann, Seefeld

\section{Antihistaminikum in neuer Galenik}

Das nicht sedierende Antihistaminikum Aerius ${ }^{\circledR}$, Wirkstoff Desloratadin, ist seit September auch als Schmelztablette erhältlich. Die Tablette löst sich im Mund innerhalb von Sekunden auf und hinterlässt einen angenehm fruchtigen Geschmack. Da dazu kein Wasser erforderlich ist, kann der Patient Ort und Zeit der Medikation frei bestimmen. Eine Einnahme einmal täglich reicht dabei aus, die Wirkung hält über 24 Stunden an. Die neue Schmelztablette ergänzt die bisherigen Darreichungsformen von Aerius ${ }^{\circledR}$ als Filmtabletten und als Sirup und ist in zwei Dosierungen erhältlich: Als Tablette mit 2,5 mg Desloratadin ist sie für Kinder von sechs bis elf Jahren zugelassen, als Tablette mit $5 \mathrm{mg}$ Desloratadin für Erwachsene und Kinder ab zwölf Jahren, jeweils zur Behandlung von allergischen Rhinitis und chronischer idiopathischer Urtikaria.

Nach Informationen von essex pharma, München

\title{
Fixkombination unter neuem Dach
}

D as Asthmapräparat Inuvair ${ }^{\circledR}$, eine fixe Kombination der Wirkstoffe Formoterol und Beclometason, erweitert seit 1. September die Produktpalette von Janssen-Cilag. Die Übernahme von Inuvair ${ }^{\circledR}$, das in Deutschland seit Oktober 2006 auf dem Markt ist, markiert den Einstieg des Neusser Pharmaunternehmens in den Asthmabereich.

Beide Wirkstoffe liegen bei Inuvair ${ }^{\circledR}$ - anders als bei herkömmlichen Dosieraerosolen, die Suspensionen enthalten gelöst in dem für die Ozonschicht unschädlichen Treibgas Norfluran vor. Dank der homogenen Lösung kann die Düse des Gerätes sehr klein gewählt werden, was eine besonders alveolengängige Aerosolwolke zur Folge hat. Diese entsteht über einen längeren Zeitraum und bewegt sich nur langsam. Das vermin- dert die oropharyngeale Deposition und erleichtert dem Patienten die Koordination während der Einatmung. Ein gleichmäßiges Vordringen der Wirkstoffe bis in die Lungenperipherie ist möglich - unabhängig vom Inspirationsfluss.

Durch diese innovative Inhalationstechnik kann bei Inuvair ${ }^{\circledR}$ die erforderliche Glukokortikoiddosis im Vergleich zu den früheren FCKW-Dosieraerosolen, die Beclometason als Monosubstanz enthielten, um rund $60 \%$ gesenkt werden. Seine Wirksamkeit und gute Verträglichkeit hat Inuvair $^{\circledR}$ in mehreren klinischen Studien unter Beweis gestellt.

Nach Informationen von Janssen-Cilag, Neuss

\section{Individuelle Therapie bei Neurodermitis}

$\mathrm{T}$ opische Calcineurininhibitoren wie Tacrolimus-Salbe (Protopic ${ }^{\circledR}$ ) sind seit 2002 in Europa zur Behandlung der atopischen Dermatitis zugelassen. Einer der ersten europäischen Experten, die mit Tacrolimus arbeiteten, war Prof. Dr. Agustin Alomar, Barcelona, Spanien. Er sieht große Vorteile in der Verwendung des Wirkstoffs, etwa das Fehlen der typischen Nebenwirkungen von Glukokortikoiden. Tacrolimus-Salbe lässt sich problemlos auch im Gesichtsbereich verwenden. Sie ist auch eine Option für Patienten mit Glukokortikoidunverträglichkeit. Bei Tachyphylaxie oder wiederholten Rebounds nach Unterbrechungen einer Glukokortikoidtherapie sollte unbedingt ein Behandlungsversuch mit Tacrolimus unternommen werden, empfahl Alomar.

Prof. Dr. Thomas Ruzicka, München, bezeichnete die topischen Calcineurininhibitoren als die wichtigste Neuentwicklung für die Dermatologie in den letzten zehn bis fünfzehn Jahren. Über 5,4 Mio. Verordnungen von Protopic $^{\circledR}$ weltweit sprächen für sich. In der Therapie empfahl Ruzicka eine indivi- duelle Vorgehensweise, bei der alle möglichen Krankheitsauslöser zu beachten seien: Ernährung, Stress, Umgebungseinflüsse, Immuntherapie etc. Gerade bei Kindern müsste man unbedingt auch die Wünsche und Ängste der Eltern berücksichtigen.

Zudem ist die persönliche Information und Schulung der Ekzempatienten sehr wichtig. Denn sie trägt nachweislich zu einer verbesserten Compliance bei. Hier setzt auch das versuchsweise in nordeuropäischen Ländern durchgeführte Programm „Astellas Skin Sense“ an, bei dem Patienten über zwei Monate von geschulten Krankenschwestern beraten werden. Informationsmaterialien klären über die wichtigsten Sachverhalte und Hintergründe auf. Der Erfolg dieses Programms soll jetzt in einer Studie untersucht werden.

Satellitensymposium „Eczema treatment: what makes skin sense?". 16th Congress of the European Academy of Dermatology and Venerology, Wien, Österreich, 16.-20. Mai 2007. Veranstalter: Astellas Pharma, München 